\title{
Primary capture of high molecular weight nucleic acids using aqueous two-phase systems
}

\begin{abstract}
The practical feasibility and generic applicability of the selective partition of plasmid DNA (pDNA) and RNA in polyethylene glycol 300 (PEG-300) and di-potassium hydrogen phosphate aqueous two-phase systems (ATPS) by addition of $\mathrm{NaCl}$ salt was investigated with partially disrupted Escherichia coli (E. coli) cell paste. The process yielded a relatively protein-free pDNA solution with $89.5 \%$ pDNA recovery while low molecular weight RNA was partially removed. The results gained here led the way to an alternative process options for the processing of pDNA. A reproducible process involving two extraction steps to recover pDNA from partially disrupted E. coli cell paste was developed and is presented here.
\end{abstract}

Keyword: Selective partition; Plasmid DNA; ATPS; NaCl; E. coli cell paste 